J. Dairy Sci. 104:6327-6327

https://doi.org/10.3168/jds.2021-104-5-6327

(c) 2021 American Dairy Science Association ${ }^{\circledR}$. Published by Elsevier Inc. and Fass Inc. All rights reserved.

\title{
Corrigendum to "Relationships between metabolic profiles and gene expression in liver and leukocytes of dairy cows in early lactation" (J. Dairy Sci. 104:3596-3616)
}

\author{
D. C. Wathes, Z. Cheng, M. Salavati, L. Buggiotti, H. Takeda, L. Tang, F. Becker, K. L. Ingvartsen, C. Ferris, \\ M. Hostens, M. A. Crowe, and the GplusE Consortium
}

On the title page (page 3596), the eighth author's initials were incorrect; the correct name is K. L. Ingvartsen. In the title page footnote, 3 authors were inadvertently omitted from the GplusE Consortium. The corrected listing is given here (corrections in bold):

Authors in the GplusE Consortium: Niamh McLoughlin, Alan Fahey, Elizabeth Matthews, Andreia Santoro, Colin Byrne, Pauline Rudd, Roisin O'Flaherty, Sinead Hallinan, Claire Wathes, Zhangrui Cheng, Ali Fouladi, Geoff Pollott, Dirk Werling, Beatriz Sanz Bernardo, Mazdak Salavati, Laura Buggiotti, Alistair Wylie, Matt Bell, Mieke Vaneetvelde, Kristof Hermans, Geert Opsomer, Sander Moerman, Jenne De Koster, Hannes Bogaert, Jan Vandepitte, Leila Vandevelde, Bonny Vanranst, Johanna Hoglund, Susanne Dahl, Soren Ostergaard, Janne Rothmann, Mogens Krogh, Else Meyer, Charlotte Gaillard, Jehan Ettema, Tine Rousing, Federica Signorelli, Francesco Napolitano, Bianca Moioli, Alessandra Crisa, Luca Buttazzoni, Jennifer McClure, Daragh Matthews, Francis Kearney, Andrew Cromie, Matt McClure, Shujun Zhang, Xing Chen, Huanchun Chen, Junlong Zhao, Liguo Yang, Guohua Hua, Chen Tan, Guiqiang Wang, Michel Bonneau, Andrea Pompozzi, Armin Pearn, Arnold Evertson, Linda Kosten, Anders Fogh, Thomas Andersen, Matthew Lucy, Chris Elsik, Gavin Conant, Jerry Taylor, Nicolas Gengler, Michel Georges, Frederic Colinet, Marilou Ramos Pamplona, Hedi Hammami, Catherine Bastin, Haruko Takeda, Aurelie Laine, Anne-Sophie Van Laere, Martin Schulze, Cinzia Marchitelli, Frank Becker, Sergio Palma Vera, Klaus Lønne Ingvartsen, Martin Tang Sørensen, and Leslie Foldager.

The authors regret the errors.

\section{REFERENCES}

Wathes, D. C., Z. Cheng, M. Salavati, L. Buggiotti, H. Takeda, L. Tang, F. Becker, K. L. Ingvartsen, C. Ferris, M. Hostens, and M. A. Crowe. the GplusE Consortium. 2021. Relationships between metabolic profiles and gene expression in liver and leukocytes of dairy cows in early lactation. J. Dairy Sci. 104(3):3596-3616. https://doi.org/10.3168/jds.2020-19165. 